\title{
Enhancing the Detection of Natural Thermal Entanglement with Disorder
}

\author{
Jenny Hide ${ }^{1}$, Wonmin Son ${ }^{1,2}$ and Vlatko Vedral ${ }^{1,2,3}$ \\ Department of Physics and Astronomy, EC Stoner Building, University of Leeds, Leeds, LS2 9JT, UK ${ }^{1}$ \\ Centre for Quantum Technologies, National University of Singapore, 3 Science Drive 2, Singapore 1175432 \\ Department of Physics, National University of Singapore, 2 Science Drive 3, Singapore $117542^{3}$.
}

(Dated: November 20, 2018)

\begin{abstract}
Physical systems have some degree of disorder present in them. We discuss how to treat natural, thermal entanglement in any random macroscopic system from which a thermodynamic witness bounded by a constant can be found. We propose that functional many-body perturbation theory be applied to allow either a quenched or an annealed average over the disorder to be taken. We find when considering the example of an $X X$ Heisenberg spin chain with a random coupling strength, that the region of natural entanglement detected by both witnesses can be enhanced by the disorder.

PACS numbers: 03.67.-a 05.40.-a 61.43.-j
\end{abstract}

Many-body systems have been of great interest to the condensed matter community for numerous years. More recently, ideas from quantum information have been applied to such systems, allowing new methods of quantifying their properties to be utilised. Entanglement in many-body systems, [1] is of particular significance due both to fundamental interest and applications in quantum computation. Approaching the thermodynamic limit and at finite temperatures, this natural entanglement becomes relevant in real, macroscopic systems [2].

Although entanglement between any two qubits, or even subsystems, in a large system can be (comparatively) easily calculated, quantifying entanglement between more qubits is much more complex [1]. However, using an entanglement witness we can still find critical values of the system's parameters below which the system is entangled. An entanglement witness is an observable whose expectation value is bounded for any separable state. Using a witness is advantageous as we can detect natural, and therefore thermal, entanglement of all classes while other measures detect only bipartite entanglement and/or can be used only at zero temperature.

When an entanglement witness can be derived from thermodynamic quantities, in particular the partition function, we refer to it as a thermodynamic witness [3]. If a system contains some randomness, an average over this disorder must be performed. The method used to calculate the average is dependent on the time taken to measure the witness in an experimental situation, giving either a quenched or an annealed average [4]. For each type of average, the witness will detect a different region of entanglement. It is therefore important to distinguish which we are able to measure experimentally. Previous research into average entanglement [ [5, 6, 7] has concentrated on spin chains and used sampling, which corresponds to calculating a quenched average numerically, or a renormalisation group approach to calculate how the randomness in the chain affects entanglement. These methods cannot take the differences between quenched and annealed entanglement into account. In addition, these papers look at zero temperature, bipartite entanglement. It is both in these areas, and by introducing a perturbative technique to deal with disorder in the context of entanglement, that our work offers new insight.

This letter studies natural entanglement in random systems. After averaging over the disorder, the regions of entanglement detected by the quenched and annealed witnesses can be related by Jensen's inequality [8]. As these averages are difficult to perform on a large system, we introduce the concept of using the well established many-body perturbation theory to calculate the thermodynamic entanglement witness. This method is very general and can be used, at least numerically, for any system.

To exemplify how these different averages affect a system's entanglement, we consider an $X X$ spin half chain in a uniform magnetic field with an extra random coupling strength. This system in particular is used as the majority of the calculation can be done analytically rather than numerically. Hence we calculate both the quenched and annealed witnesses for this system using many-body perturbation theory and plot the results. We find that the randomness can both enhance and create regions of entanglement detected by both witnesses. We stress that it is the region of entanglement detected by the witness that is enhanced by the disorder, rather than, necessarily, the entanglement itself. Intuitively however, increasing the disorder can increase the entanglement itself, hence we discuss possible mechanisms for this.

In order to calculate an entanglement witness in a random system, we must perform an average over the disorder. As discussed above, the appropriate average to use is dependent on how fast the measurement process is in comparison to the speed of the fluctuations of the variable in the system. As different experimental conditions require different averages to be taken of the system, it is important to determine which is used.

First we consider the possibility that we can measure each state of the system faster than the disorder fluctuates. This is called a quenched average. To calculate this, we first perform a thermal average, and then average over 
the random variable. Rather than calculating the properties of the system, we calculate an average of all possible systems. Otherwise, if the system fluctuates faster than we can measure it, we cannot measure each state the system takes, and we should use an annealed average. Here, the system thermalises before we can measure the state; hence the average over the disorder and the thermal average are taken at the same time. Experimental studies on high temperature superconducting materials discuss situations when each of these averages occur [9].

As each type of average relates to a different standard of measurement, each will give a different region of entanglement. It is therefore important to clarify which is needed when measuring a system. The regions of entanglement detected by the quenched and annealed averages can be related by invoking Jensen's inequality, $\langle f(X)\rangle \leq f\langle X\rangle$, where is $f$ is a concave function acting on $X$ and the average, $\langle\cdots\rangle$ is over the disorder. For a convex function, the inequality is reversed. The left hand side of the inequality is the quenched average, and the right hand side is the annealed average. We are interested in how these averages can change the amount of entanglement we can measure, hence we must consider how we can use entanglement witnesses in this context.

For any system of finite dimension for which we can calculate a thermodynamic witness with some constant value, $\alpha$, as the bound for detecting entanglement, we can apply the following argument. If the witness, $W$, is a convex function, the entangled region detected which we define as $E=\alpha-W$ is always concave. Similarly, $E$ is convex if the witness is concave. Hence the application of Jensen's inequality to either scenario tells us which average allows us to detect the most entanglement.

Although entanglement has previously been studied using thermodynamic witnesses calculated from the partition function of the system, to discover how disorder affects thermal, macroscopic (natural) entanglement, we instead use functional many-body perturbation theory.

We set out an outline of the method for a fermionic system below. Due to the Jordan-Wigner transformation, this also applies to any spin half system. Bosonic systems can also be studied using this formalism, but complex numbers are used in place of Grassmann variables. We first define a generating functional, $\left.Z[\bar{\eta}, \eta]\right|_{\bar{\eta}, \eta=0}=Z$ where $Z=\operatorname{Tr}\left(e^{-\beta H}\right)$ is the partition function of the system and $\eta$ is a Grassmann variable. $Z[\bar{\eta}, \eta]$ allows us to calculate expectation values using functional derivatives from an appropriate generating function, $g_{f}$ :

$$
Z[\bar{\eta}, \eta]=\operatorname{Tr}\left(e^{-\beta H} T e^{g_{f}[\bar{\eta}, \eta]}\right)
$$

where $\beta$ is the inverse temperature, $H=H_{0}+H_{1}\left(v_{l}\right)$ is the Hamiltonian of the system and $v_{l}$ is a random variable, with $l$ labelling position. $T$ is the time ordering operator. After putting (10) into path integral representation [10], we can take an average over the random variable of $Z[\bar{\eta}, \eta]^{n}$. For clarity, we consider the random variables to be taken from a Gaussian distribution.

$$
\left\langle Z^{n}[\bar{\eta}, \eta]\right\rangle=\frac{1}{(2 \pi \Delta)^{\frac{N}{2}}} \int_{-\infty}^{\infty} \prod_{l=1}^{N} d v_{l} e^{-\frac{1}{2 \Delta} \sum_{l} v_{l}^{2}} \prod_{a=1}^{n} Z\left[\bar{\eta}_{a}, \eta_{a}\right]
$$

where $\Delta$ is the variance. After calculating this average, we perform transformations to diagonalise $H_{0}$. The average can then be rewritten as

$$
\left\langle Z^{n}[\bar{\eta}, \eta]\right\rangle=Z_{0}^{n} e^{\Delta \tilde{S}_{i n t}^{n}\left[\frac{\delta}{\delta \bar{\eta}}, \frac{\delta}{\delta \eta}\right]} e^{g_{F}[\bar{\eta}, \eta]}
$$

where $Z_{0}$ is the partition function of the unperturbed system, $\Delta \tilde{S}_{i n t}^{n}\left[\frac{\delta}{\delta \bar{\eta}}, \frac{\delta}{\delta \eta}\right]$ is the perturbed term, and acts on $g_{F}[\bar{\eta}, \eta]$, the generator re-written in terms of a Greens function. Expanding $e^{\Delta \tilde{S}_{i n t}^{n}}$, we can use Feynman diagrams to calculate $\left\langle Z^{n}[\bar{\eta}, \eta]\right\rangle$. Functional derivatives can then be used to calculate an entanglement witness for both the quenched and the annealed averages. We can then implement the replica trick, $\langle\ln Z\rangle=$ $\lim _{n \rightarrow 0} \frac{1}{n}\left(\left\langle Z^{n}\right\rangle-1\right)$ in order to calculate the quenched witness. The annealed average is found simply by setting $n=1$. The replica trick corresponds to considering $n$ identical, non-interacting replicas of the system. Performing an average over the disorder of these replicas allows us to use $\Delta$, the variance rather than a different random variable at each $l$. Rather than considering the experimental situation of measuring the system many times and performing an average, the replica trick allows us to instead use many copies of the same system.

Perturbation theory is valid when the matrix elements, $\left\langle m\left|H_{1}\left(v_{l}\right)\right| n\right\rangle$, of the interaction term, $H_{1}\left(v_{l}\right)$, where $|n\rangle$ and $|m\rangle(m \neq n)$ are possible eigenvectors of $H_{0}$, are much smaller than the energy difference between $n$ and $m$. To ensure this condition is satisfied, we require $v_{l} \ll 1$ with high probability, and hence $\Delta \ll 1$.

To make the arguments above explicit, we calculate the average witness for a specific, predominantly analytically solvable, example: an $X X$ spin half chain with a random coupling strength. The unperturbed Hamiltonian is

$$
H_{0}=-\sum_{l=1}^{N}\left[\frac{J}{2}\left(\sigma_{l}^{x} \sigma_{l+1}^{x}+\sigma_{l}^{y} \sigma_{l+1}^{y}\right)+B \sigma_{l}^{z}\right]
$$

where $J$ is the coupling strength between nearest neighbours, and $B$ is a uniform magnetic field. Considering the thermodynamic limit, $N \rightarrow \infty$, the spin chain can be diagonalised [11] using a Jordan-Wigner transformation $a_{l}=\prod_{m=1}^{l-1} \sigma_{m}^{z} \otimes\left(\sigma_{l}^{x}+i \sigma_{l}^{y}\right) / 2$ and a Fourier transform, $a_{l}=\int_{-\pi}^{\pi} \frac{d q}{2 \pi} e^{i q l} d(q)$ giving $H_{0}=\int_{-\pi}^{\pi} \frac{d q}{\pi}(J \cos q-$ $B) d^{\dagger}(q) d(q)$. The partition function is then

$$
\ln Z_{0}=\frac{N}{2 \pi} \int_{-\pi}^{\pi} d q \ln (2 \cosh [\beta(J \cos q-B)])
$$




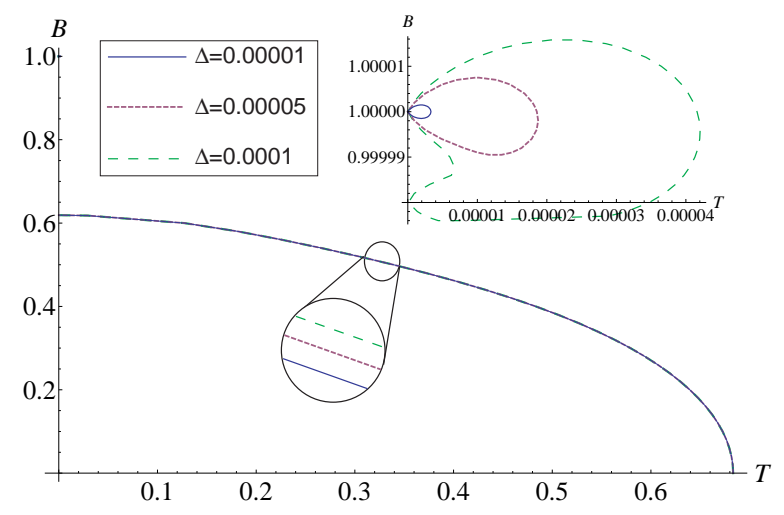

FIG. 1: This plot shows the values of uniform magnetic field, $B$ and temperature, $T$, with $J=1$ below which entanglement is detected by the quenched witness for various values of the disorder, $\Delta$. Details of this plot are discussed in the text.

We now introduce the Hamiltonian $H=H_{0}+H_{1}\left(v_{l}\right)$ and consider $H_{1}\left(j_{l}\right)=-\frac{1}{2} \sum_{l=1}^{N-1} j_{l}\left(\sigma_{l}^{x} \sigma_{l+1}^{x}+\sigma_{l}^{y} \sigma_{l+1}^{y}\right)$ where $j_{l}$ is a site dependent random coupling strength between nearest neighbours. Each random variable is taken from a Gaussian distribution centred at zero with variance $\Delta$.

To detect entanglement, we use an entanglement witness [12] which is proportional to the sum of the $x$ and $y$ components of the susceptibility, and is therefore measurable in practice [13].

$$
W=\left|\frac{2}{\beta N} \frac{\partial}{\partial J} \ln Z\right|=\left|\frac{1}{N} \sum_{l}\left\langle\sigma_{l}^{x} \sigma_{l+1}^{x}+\sigma_{l}^{y} \sigma_{l+1}^{y}\right\rangle\right|
$$

The system is entangled when the witness is greater than 1. This bound is found using the Cauchy-Schwarz inequality and properties of the density matrix [14, 15].

The entangled region, $E=\alpha-W$, is concave as $W$ is always convex due to the absolute sign. Using Jensen's inequality, this means the region of entanglement detected by the quenched witness is equal to or less than the region detected by the annealed witness.

In order to use functional many-body perturbation theory, we must first define an appropriate generating function with which to calculate the witness. Letting $g_{f}[\bar{\eta}, \eta]=\int_{0}^{\beta} d \tau \sum_{l}\left[\bar{\eta}_{l}(\tau) a_{l}(\tau)+\eta_{l}(\tau) a_{l}^{\dagger}(\tau)\right]$ in (11) and diagonalising the witness,

$$
W=\left|\frac{4}{N} \int_{-\pi}^{\pi} \frac{d q}{2 \pi} \cos q\left\langle d^{\dagger}(q) d(q)\right\rangle\right|
$$

we find $\left\langle d^{\dagger}(q) d(q)\right\rangle=\lim _{n \rightarrow x} \frac{\delta}{\delta \eta_{b}\left(\tau^{\prime}, q^{\prime}\right)} \frac{\delta}{\delta \bar{\eta}_{a}(\tau, q)}\left\langle Z^{n}[\bar{\eta}, \eta]\right\rangle$ with $x=0$ for the quenched average and $x=1$ for the annealed average.

Considering $H_{1}\left(j_{l}\right)$, we average over $j_{l}$ in (2) and calculate equation (3) from $\ln Z_{0}$ as given in (5), $\Delta \tilde{S}_{i n t}^{n}=$

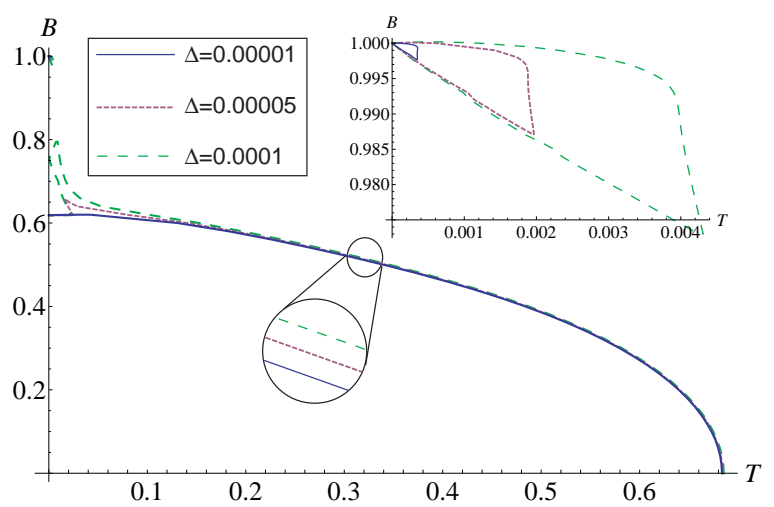

FIG. 2: This plot shows the values of uniform magnetic field, $B$ and temperature, $T$, with $J=1$ below which entanglement is detected by the annealed witness for various values of the disorder, $\Delta$. Details of this plot are discussed in the text.

$\frac{1}{2} \int_{0}^{\beta} d \tau d \tau^{\prime} \int_{-\pi}^{\pi} \Pi_{j=1}^{4} \frac{d q_{j}}{2 \pi} \sum_{a b c d} \delta_{a b} \delta_{c d} 2 \pi \delta\left(q_{1}-q_{2}+q_{3}-\right.$ $\left.q_{4}\right)\left(e^{i q_{1}}+e^{-i q_{2}}\right)\left(e^{i q_{3}}+e^{-i q_{4}}\right)\left[2 \pi \frac{\delta}{\delta \bar{\eta}_{b}\left(\tau, q_{2}\right)} 2 \pi \frac{\delta}{\delta \eta_{a}\left(\tau, q_{1}\right)}\right]$. $\left[2 \pi \frac{\delta}{\delta \bar{\eta}_{d}\left(\tau^{\prime}, q_{4}\right)} 2 \pi \frac{\delta}{\delta \eta_{c}\left(\tau^{\prime}, q_{3}\right)}\right]$, and the source term, $g_{F}[\bar{\eta}, \eta]=$ $\int_{0}^{\beta} d \tau d \tau^{\prime} \int_{-\pi}^{\pi} \frac{d q}{2 \pi} \sum_{a b} \bar{\eta}_{a}(\tau, q) G_{a b}\left(\tau-\tau^{\prime}, q\right) \eta_{b}\left(\tau^{\prime}, q\right)$.

After expanding $e^{\Delta \tilde{S}_{i n t}^{n}}$ and calculating Feynman diagrams to first order, we find the witness is

$$
W_{\text {ave }}=\left|\frac{2}{\pi} \int_{-\pi}^{\pi} d q \cos q\left[n(q)-\Delta G_{\text {ave }}^{(1)}\right]\right|
$$

with ave indicating either a quenched, $q$ or annealed, a average. From the method outlined above, we find that $G_{q}^{(1)}=$ $\frac{2}{\pi} \int_{-\pi}^{\pi} d p \cos ^{2}\left(\frac{q+p}{2}\right)\left[\frac{n(q)-n(p)}{(\varepsilon(p)-\varepsilon(q))^{2}}-\frac{\beta n(q)(1-n(q))}{\varepsilon(p)-\varepsilon(q)}\right], \quad$ and $G_{a}^{(1)}=G_{q}^{(1)}+\frac{2 \beta^{2}}{\pi} n(q)(1-n(q)) \cos q \int_{-\pi}^{\pi} d p n(p) \cos p$. Here, $n(x)=\left(e^{\beta \varepsilon(x)}+1\right)^{-1}$ and $\varepsilon(x)=2 J \cos x-2 B$.

As discussed previously, to ensure our use of perturbation theory is valid, we restrict the value of the disorder to $\Delta \leq 0.0001$. We note that all the features found in Figs. (11) and (2) are present even when $\Delta \ll 0.0001$.

The entanglement detected by the witness in an unperturbed $X X$ spin half chain, plotted in [12] (Fig. (1) at $b=0$ ), exists below a critical uniform magnetic field, $B$, and temperature, $T$, and is below the coupling strength between nearest neighbour sites, $J$. Much attention has been focused on the study of quantum phase transitions [16] and we note that $H_{0}$ has one at $B=J$. The spectrum of the unperturbed spin chain [17] at zero temperature is as follows: at $B=0$, the ground state of the spin chain is a fully symmetric state of all permutations of an equal number of up and down spins. On increasing $B$, the number of up spins increases until, when $B>J$, they are all spin up and the system is in a product state. 
Fig. (11) shows how the entanglement detected by the quenched witness behaves. Introducing the random coupling strength enhances the region detected by the witness. Increasing $\Delta$ increases the entangled region detected compared to that in the original $X X$ model. A higher variance increases the probability of a high value of $j_{l}$ (though this may be positive or negative) being picked out of the Gaussian distribution. As we consider small $\Delta$ and a larger coupling strength increases entanglement, so does increasing $\Delta$. Further, a region of entanglement close to $B=J$ is created at low temperatures which again increases with $\Delta$. We note that this extra region of entanglement is created at the quantum phase transition of $H_{0}$. The annealed witness is plotted in Fig. (2) and gives a larger entangled region than the quenched witness everywhere except at $T=0$ where they coincide. Hence the plots demonstrate the importance of an accurate determination of how we measure the system experimentally. The behaviour of the plot as $\Delta$ changes remains the same as for the quenched witness.

Alternatively, we can view the system in the following way: even if the ground state of a system is separable, there is a possibility of creating entanglement by increasing the temperature [18]. A finite temperature allows higher energy levels to mix with the ground state. If these newly available energy levels are themselves entangled, on mixing with a separable ground state, entanglement can be created and/or increase. Similarly, introducing disorder into a system allows higher energy levels to be populated even at zero temperature, allowing for the possibility of creating or enhancing entanglement.

We have also considered the effect of a random magnetic field, $b_{l}$ on the witness. The plots for the quenched and the annealed witness have similar features as for $j_{l}$. The region of entanglement close to $B=J$ increases with $\Delta$ similarly to the random coupling strength plots. At lower $B$, increasing $\Delta$ decreases the entangled region, contrary to the random coupling strength case. A random magnetic field increases the chance of spin flip events. For lower $B$ this can only act to decrease the entanglement due to the highly entangled structure of the ground state of $H_{0}$. At higher $B$, particularly at $B=J$, this same effect will increase the entanglement due to the almost separable ground state at this point.

This letter has studied entanglement in the limit of small disorder. The opposite, large disorder limit is also of interest; for $b_{l}$, it is clear that large disorder would cause the system to become separable as $H \rightarrow \sum b_{l} \sigma_{l}^{z}$. However, the case for $j_{l}$ is much more complex. Using the path integral formalism, we can consider $H_{1}\left(j_{l}\right)$ to be the unperturbed part of the Hamiltonian, and $H_{0}$ to be the perturbation. As this cannot be done analytically, we do not pursue this here. We do, however, still expect that in the limit of large disorder the system should become completely mixed, and hence separable, though the effect of averaging over this disorder on the behaviour of the witness is nontrivial. This uncertainty is due to the equally high probability of a large positive, and hence entanglement enhancing, or negative, and hence entanglement destroying, value of $j_{l}$. While beyond the scope of this letter, this would be an interesting topic to study.

We have discussed how the quenched and annealed entanglement witnesses are related both experimentally and via Jensen's inequality, and found that we can apply functional many-body perturbation theory to a random system in order to calculate these witnesses. We have found that randomness in a system can enhance the entanglement detected by a witness. In particular, we have considered a spin chain with a random coupling strength. This result is important in the experimental realisation of quantum systems due to entanglement being a resource in quantum computation as it demonstrates that disorder may actually be beneficial even in thermal systems.

Acknowledgements - We are very grateful to I. Lawrie for many extensive discussions on issues related to this letter. V.V. and J.H. acknowledge the EPSRC for financial support. V.V. is grateful for funding from the Wolfson Foundation, the Royal Society and the E.U. His work is also supported by the National Research Foundation (Singapore) and the Ministry of Education (Singapore).

[1] L. Amico, R. Fazio, A. Osterloh and V. Vedral, Rev. Mod. Phys. 80, 517 (2008)

[2] V. Vedral, Nature, 453, 1004 (2008)

[3] M. R. Dowling, A. C. Doherty and S. D. Bartlett, Phys. Rev. A, 70, 062113 (2004)

[4] C. De Dominicis and I. Giardina, Random Fields and Spin Glasses: A Field Theory Approach, Cambridge University Press, Cambridge, 2006

[5] G. De Chiara, S. Montangero, P. Calabrese and R. Fazio, J. Stat. Mech. P03001 (2006)

[6] M. Fujinaga and N. Hatano, J. Phys. Soc. Jpn. 76, 094001 (2007)

[7] D. Binosi, G. De Chiara, S. Montangero and A. Recati, Phys. Rev. B, 76, 140405(R) (2007)

[8] J. L. W. V. Jensen, Acta Math. 30, 175 (1906)

[9] B. O. Wells et al., Science, 277, 1067 (1997)

[10] B. F. Hatfield, Quantum Field Theory of Point Particles and Strings, Westview Press, USA, 1992

[11] S. Katsura, Phys. Rev. 127, 1508 (1962)

[12] J. Hide, W. Son, I. Lawrie and V. Vedral, Phys. Rev. A, 76, 022319 (2007)

[13] C. Brukner, V. Vedral and A. Zeilinger, Phys. Rev. A 73, $012110(2006)$

[14] G. Toth, Phys. Rev. A 71, 010301(R) (2005)

[15] C. Brukner and V. Vedral, arXiv:quant-ph/0406040 (2004)

[16] A. Osterloh, L. Amico, G. Falci and R. Fazio, Nature, 416, 608 (2002)

[17] W. Son, L. Amico, F. Plastina and V. Vedral, arxiv: $0807.1602(2008)$

[18] M. C. Arnesen, S. Bose and V. Vedral, Phys. Rev. Lett. 87, 017901 (2001) 